J. Case

SNMP Research, Inc.

K. MCCloghrie

Hughes LAN Systems

M. Rose

Dover Beach Consulting, Inc.

S. Waldbusser

Carnegie Mellon University

April 1993

\title{
Protocol Operations \\ for version 2 of the \\ Simple Network Management Protocol (SNMPv2)
}

\begin{abstract}
Status of this Memo
This RFC specifes an IAB standards track protocol for the Internet community, and requests discussion and suggestions for improvements. Please refer to the current edition of the "IAB Official Protocol standards" for the standardization state and status of this protocol. Distribution of this memo is unlimited.
\end{abstract}

\section{Table of Contents}

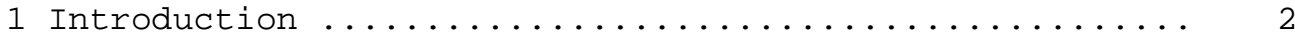

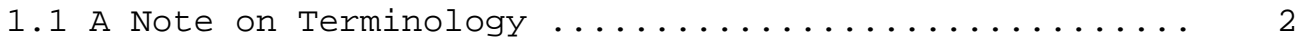

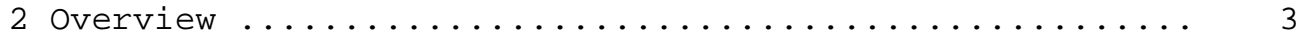

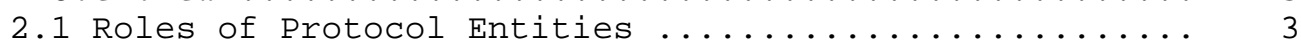

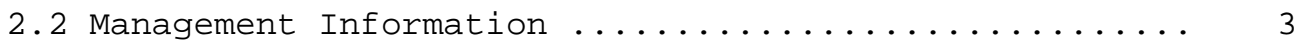

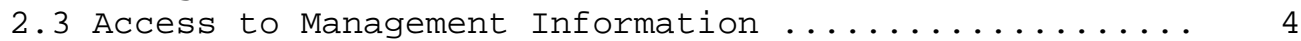

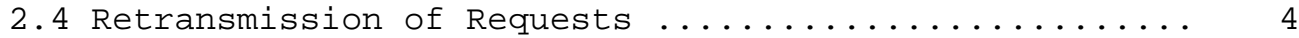

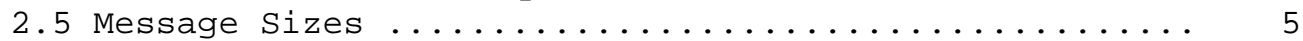

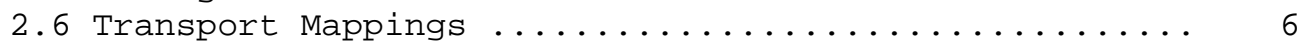

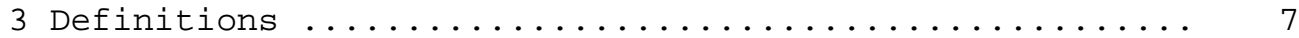

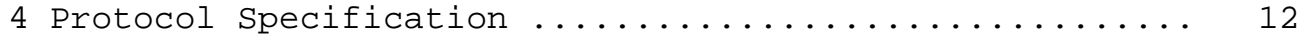

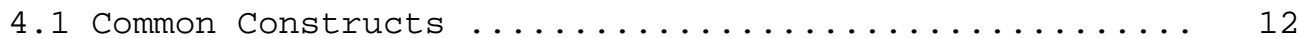

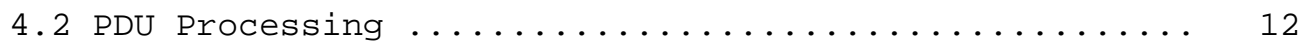

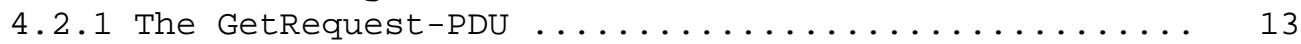

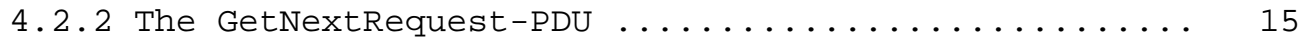

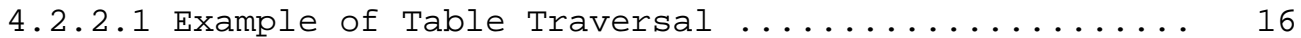

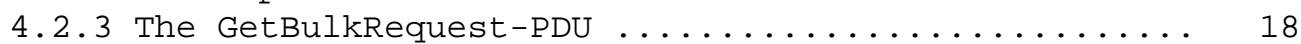

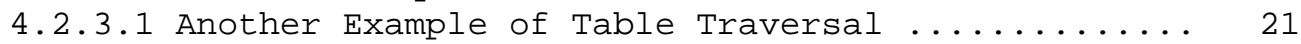

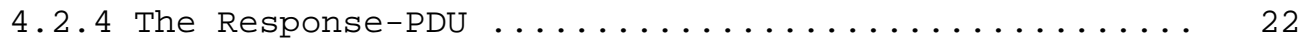

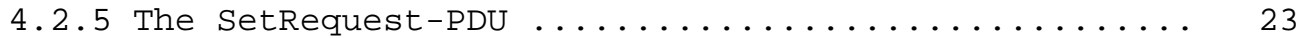

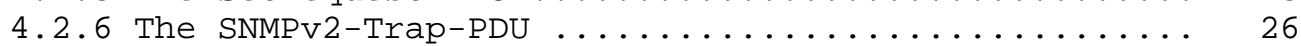

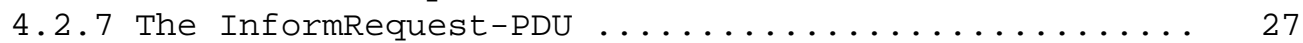




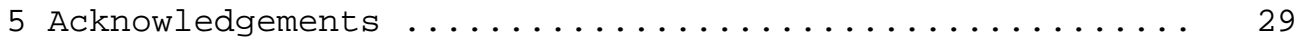

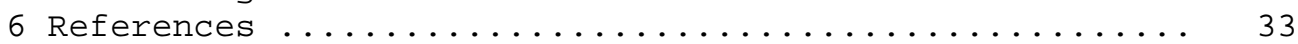

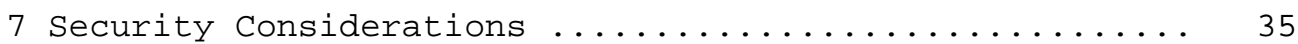

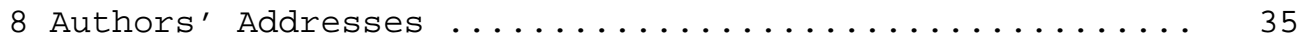




\section{Introduction}

A network management system contains: several (potentially many) nodes, each with a processing entity, termed an agent, which has access to management instrumentation; at least one management station; and, a management protocol, used to convey management information between the agents and management stations. Operations of the protocol are carried out under an administrative framework which defines both authentication and authorization policies.

Network management stations execute management applications which monitor and control network elements. Network elements are devices such as hosts, routers, terminal servers, etc., which are monitored and controlled through access to their management information.

Management information is viewed as a collection of managed objects, residing in a virtual information store, termed the Management Information Base (MIB). Collections of related objects are defined in MIB modules. These modules are written using a subset of OSI's Abstract syntax Notation One (ASN.1)

[1], termed the Structure of Management Information (SMI) [2].

The management protocol, version 2 of the Simple Network Management Protocol, provides for the exchange of messages which convey management information between the agents and the management stations. The form of these messages is a message "wrapper" which encapsulates a Protocol Data Unit (PDU). The form and meaning of the "wrapper" is determined by an administrative framework which defines both authentication and authorization policies.

It is the purpose of this document, Protocol Operations for SNMPv2, to define the operations of the protocol with respect to the sending and receiving of the PDUs.

\subsection{A Note on Terminology}

For the purpose of exposition, the original Internet-standard Network Management Framework, as described in RFCs 1155, 1157, and 1212, is termed the SNMP version 1 framework (SNMPV1). The current framework is termed the SNMP version 2 framework (SNMPV2). 


\section{Overview}

2.1. Roles of Protocol Entities

A SNMPV2 entity may operate in a manager role or an agent role.

A SNMPv2 entity acts in an agent role when it performs SNMPv2 management operations in response to received SNMPv2 protocol messages (other than an inform notification) or when it sends trap notifications.

A SNMPV2 entity acts in a manager role when it initiates SNMPV2 management operations by the generation of SNMPV2 protocol messages or when it performs SNMPV2 management operations in response to received trap or inform notifications.

A SNMPV2 entity may support either or both roles, as dictated by its implementation and configuration. Further, a SNMPv2 entity can also act in the role of a proxy agent, in which it appears to be acting in an agent role, but satisfies

management requests by acting in a manager role with a remote entity. The use of proxy agents and the transparency principle that defines their behavior is described in [3] .

\subsection{Management Information}

The term, variable, refers to an instance of a non-aggregate object type defined according to the conventions set forth in the SMI [2] or the textual conventions based on the SMI [4] . The term, variable binding, normally refers to the pairing of the name of a variable and its associated value. However, if certain kinds of exceptional conditions occur during processing of a retrieval request, a variable binding will pair a name and an indication of that exception.

A variable-binding list is a simple list of variable bindings.

The name of a variable is an OBJECT IDENTIFIER which is the concatenation of the OBJECT IDENTIFIER of the corresponding object-type together with an OBJECT IDENTIFIER fragment identifying the instance. The OBJECT IDENTIFIER of the corresponding object-type is called the OBJECT IDENTIFIER 
prefix of the variable.

\subsection{Access to Management Information}

Three types of access to management information are provided by the protocol. One type is a request-response interaction, in which a SNMPV2 entity, acting in a manager role, sends a request to a SNMPv2 entity, acting in an agent role, and the latter SNMPV2 entity then responds to the request. This type is used to retrieve or modify management information associated with the managed device.

A second type is also a request-response interaction, in which a SNMPv2 entity, acting in a manager role, sends a request to a SNMPv2 entity, also acting in a manager role, and the latter SNMPV2 entity then responds to the request. This type is used to notify a SNMPV2 entity, acting in a manager role, of management information associated with another SNMPv2 entity, also acting in a manager role.

The third type of access is an unconfirmed interaction, in which a SNMPV2 entity, acting in an agent role, sends a unsolicited message, termed a trap, to a SNMPv2 entity, acting in a manager role, and no response is returned. This type is used to notify a SNMPv2 entity, acting in a manager role, of an exceptional situation, which has resulted in changes to management information associated with the managed device.

\subsection{Retransmission of Requests}

For all types of request in this protocol, the receiver is required under normal circumstances, to generate and transmit a response to the originator of the request. Whether or not a request should be retransmitted if no corresponding response is received in an appropriate time interval, is at the discretion of the application originating the request. This will normally depend on the urgency of the request. However, such an application needs to act responsibly in respect to the frequency and duration of re-transmissions. 


\subsection{Message Sizes}

The maximum size of a SNMPV2 message is limited the minimum of :

(1) the maximum message size which the destination SNMPv2 entity can accept; and,

(2) the maximum message size which the source SNMPv2 entity can generate.

The former is indicated by partyMaxMessagesize[5] of the destination party. The latter is imposed by implementationspecific local constraints.

Each transport mapping for the SNMPV2 indicates the minimum message size which a SNMPv2 implementation must be able to produce or consume. Although implementations are encouraged to support larger values whenever possible, a conformant implementation must never generate messages larger than allowed by the receiving SNMPv2 entity.

One of the aims of the GetBulkRequest-PDU, specified in this protocol, is to minimize the number of protocol exchanges required to retrieve a large amount of management information. As such, this PDU type allows a SNMPv2 entity acting in a manager role to request that the response be as large as possible given the constraints on message sizes. These constraints include the limits on the size of messages which the SNMPv2 entity acting in an agent role can generate, and the SNMPV2 entity acting in a manager role can receive.

However, it is possible that such maximum sized messages may be larger than the Path MTU of the path across the network traversed by the messages. In this situation, such messages are subject to fragmentation. Fragmentation is generally considered to be harmful [6], since among other problems, it leads to a decrease in the reliability of the transfer of the messages. Thus, a SNMPv2 entity which sends a GetBulkRequest-PDU must take care to set its parameters accordingly, so as to reduce the risk of fragmentation. In particular, under conditions of network stress, only small values should be used for max-repetitions. 


\subsection{Transport Mappings}

It is important to note that the exchange of SNMPv2 messages requires only an unreliable datagram service, with every message being entirely and independently contained in a single transport datagram. Specific transport mappings and encoding rules are specified elsewhere [7]. However, the preferred mapping is the use of the User Datagram Protocol [8]. 
3. Definitions

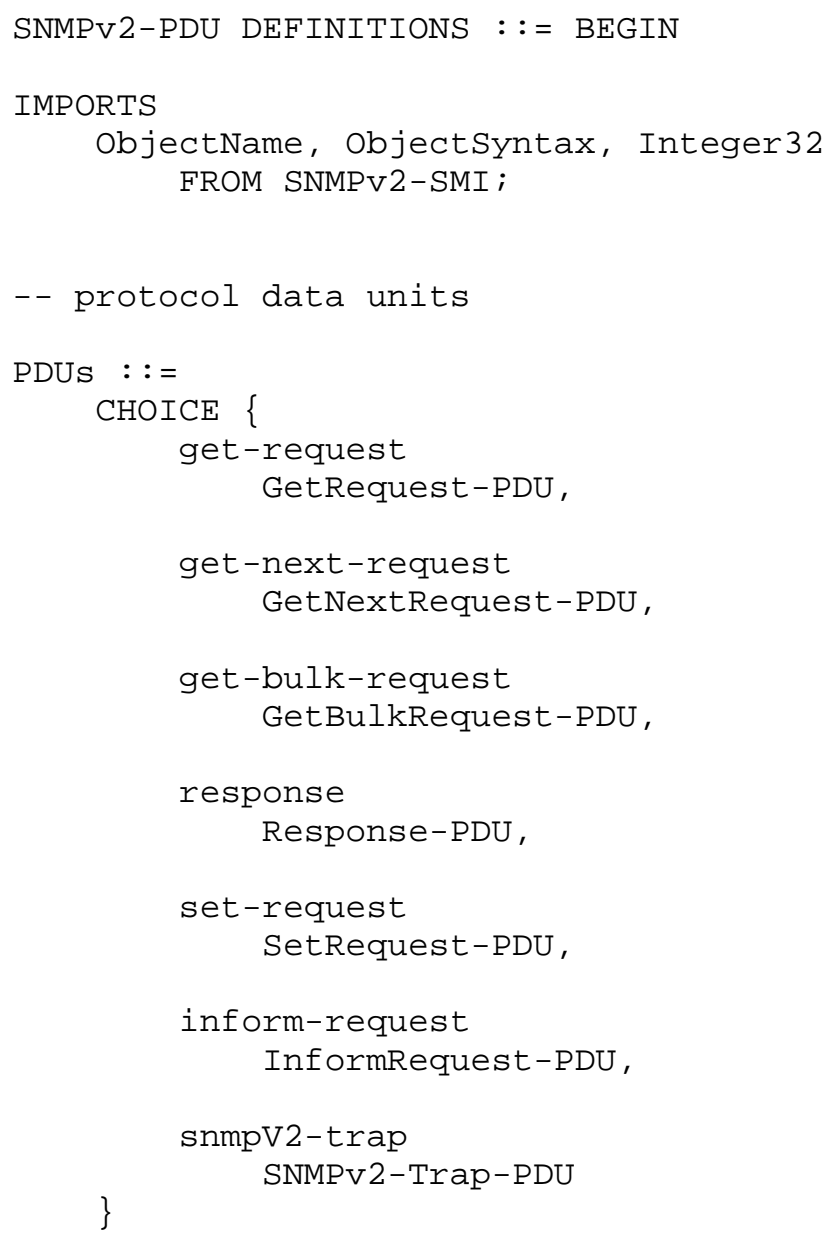




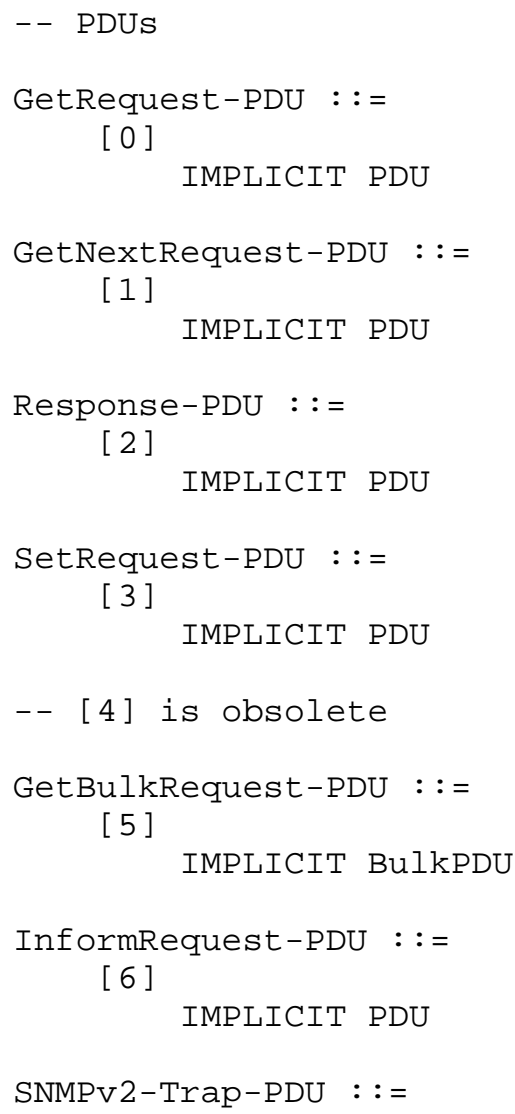




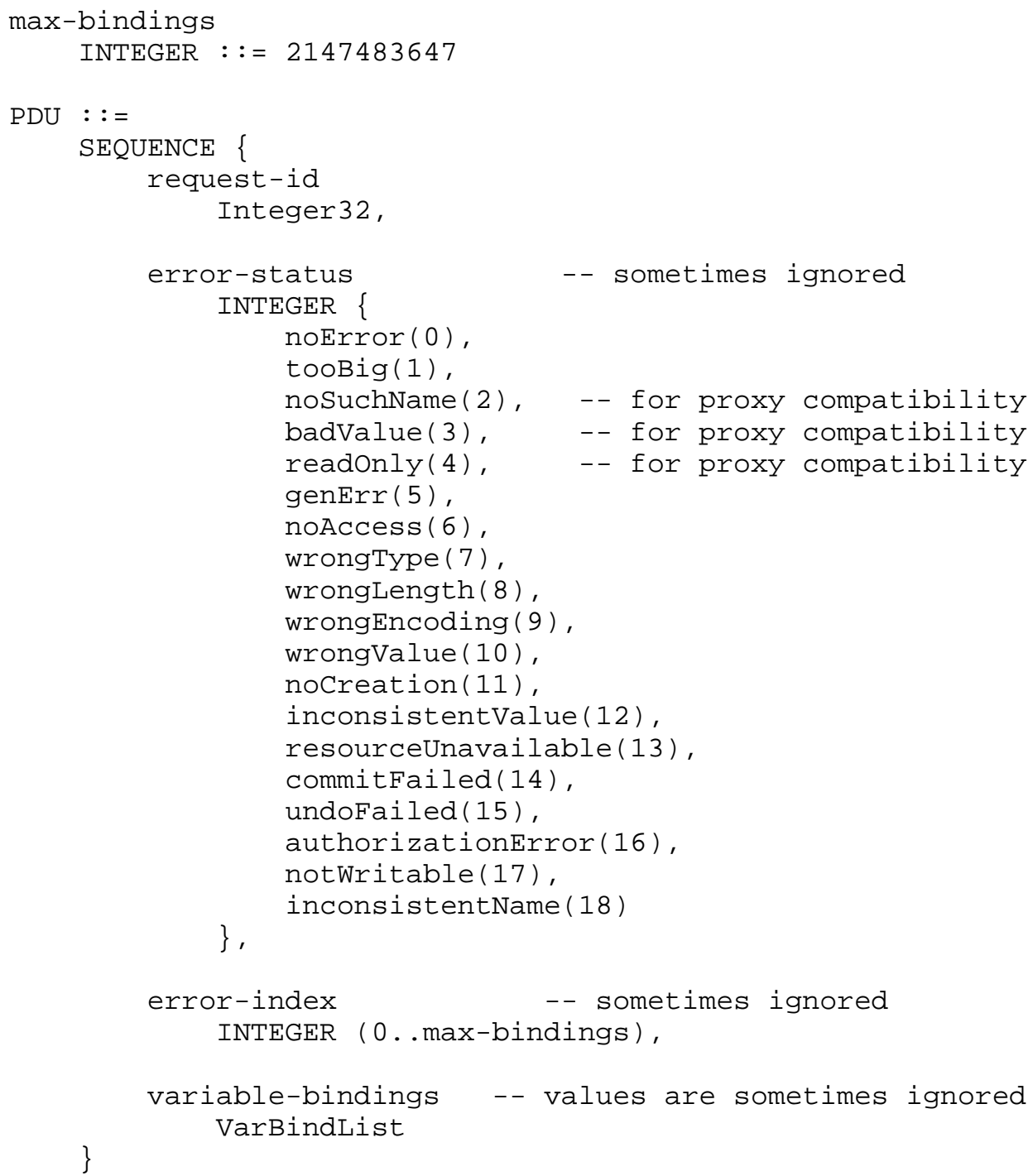




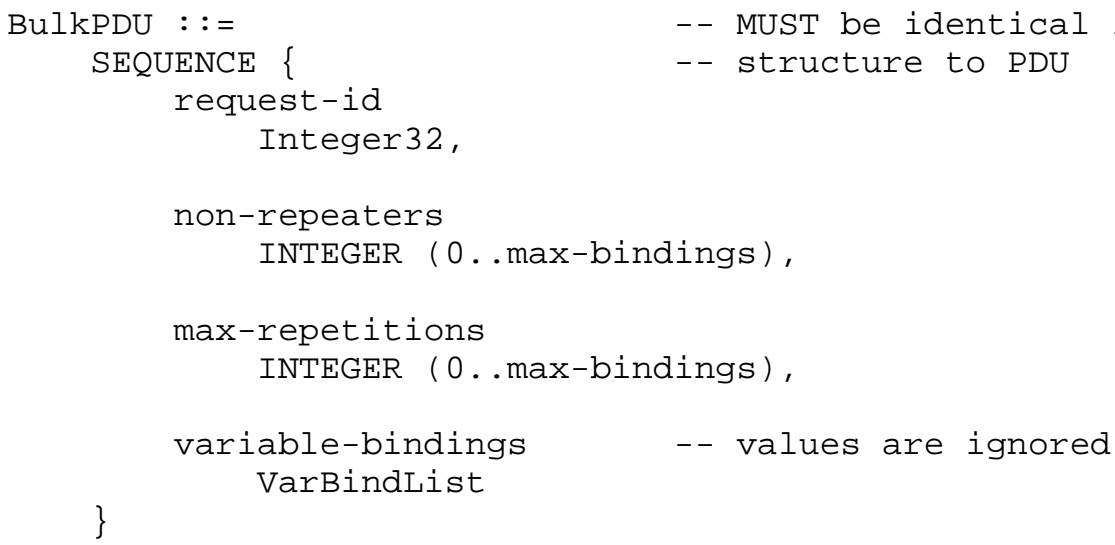




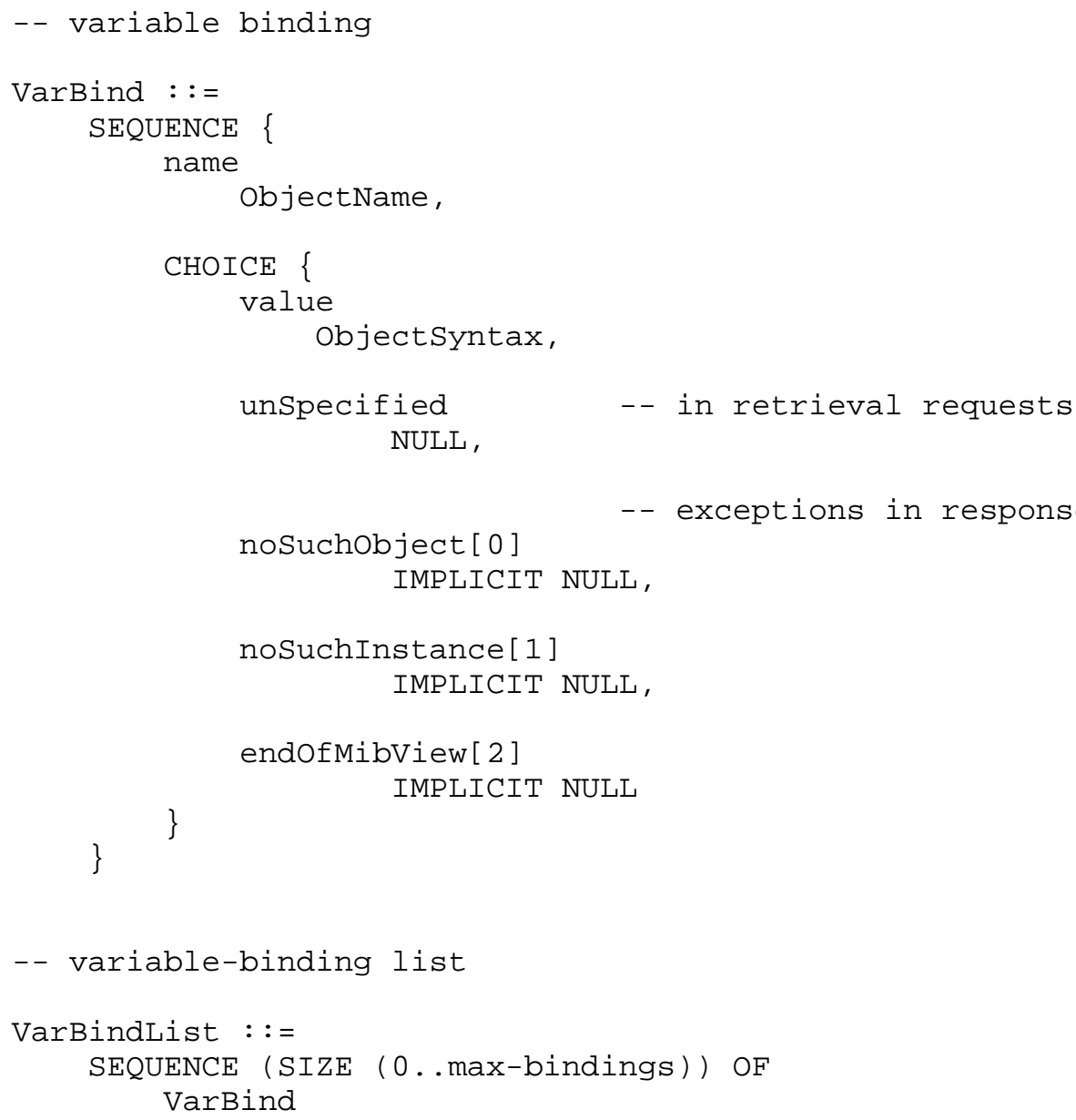

END 


\section{Protocol Specification}

\subsection{Common Constructs}

The value of the request-id field in a Response-PDU takes the value of the request-id field in the request PDU to which it is a response. By use of the request-id value, a SNMPv2 application can distinguish the (potentially multiple) outstanding requests, and thereby correlate incoming responses with outstanding requests. In cases where an unreliable datagram service is used, the request-id also provides a simple means of identifying messages duplicated by the network. Use of the same request-id on a retransmission of a request allows the response to either the original transmission or the retransmission to satisfy the request. However, in order to calculate the round trip time for transmission and processing of a request-response transaction, the SNMPV2 application needs to use a different request-id value on a retransmitted request. The latter strategy is recommended for use in the majority of situations.

A non-zero value of the error-status field in a Response-PDU is used to indicate that an exception occurred to prevent the processing of the request. In these cases, a non-zero value of the Response-PDU's error-index field provides additional information by identifying which variable binding in the list caused the exception. A variable binding is identified by its index value. The first variable binding in a variable-binding list is index one, the second is index two, etc.

SNMPV2 limits OBJECT IDENTIFIER values to a maximum of 128 sub-identifiers, where each sub-identifier has a maximum value of $2 * \star 32-1$.

\subsection{PDU Processing}

It is mandatory that all SNMPv2 entities acting in an agent role be able to generate the following PDU types: Response-PDU and SNMPv2-Trap-PDU; further, all such implementations must be able to receive the following PDU types: GetRequest-PDU, GetNextRequest-PDU, GetBulkRequest-PDU, and SetRequest-PDU. 
It is mandatory that all SNMPv2 entities acting in a manager role be able to generate the following PDU types: GetRequestPDU, GetNextRequest-PDU, GetBulkRequest-PDU, SetRequest-PDU, InformRequest-PDU, and Response-PDU; further, all such implementations must be able to receive the following PDU types: Response-PDU, SNMPv2-Trap-PDU, InformRequest-PDU;

In the elements of procedure below, any field of a PDU which is not referenced by the relevant procedure is ignored by the receiving SNMPV2 entity. However, all components of a PDU, including those whose values are ignored by the receiving SNMPv2 entity, must have valid ASN.1 syntax and encoding. For example, some PDUs (e.g., the GetRequest-PDU) are concerned only with the name of a variable and not its value. In this case, the value portion of the variable binding is ignored by the receiving SNMPv2 entity. The unspecified value is defined for use as the value portion of such bindings.

For all generated PDUs, the message "wrapper" to encapsulate the PDU is generated and transmitted as specified in [3]. The size of a message is limited only by constraints on the maximum message size, either a local limitation or the limit associated with the message's destination party, i.e., it is not limited by the number of variable bindings.

On receiving a management communication, the procedures defined in section 3.2 of [3] are followed. If these procedures indicate that the PDU contained within the message "wrapper" is to be processed, then the SNMPv2 context associated with the PDU defines the object resources which are visible to the operation.

\subsubsection{The GetRequest-PDU}

A GetRequest-PDU is generated and transmitted at the request of a SNMPv2 application.

Upon receipt of a GetRequest-PDU, the receiving SNMPv2 entity processes each variable binding in the variable-binding list to produce a Response-PDU. All fields of the Response-PDU have the same values as the corresponding fields of the received request except as indicated below. Each variable binding is processed as follows: 
(1) If the variable binding's name does not have an OBJECT IDENTIFIER prefix which exactly matches the OBJECT IDENTIFIER prefix of any variable accessible by this request, then its value field is set to 'nosuchobject'.

(2) Otherwise, if the variable binding's name does not exactly match the name of a variable accessible by this request, then its value field is set to 'noSuchInstance'.

(3) Otherwise, the variable binding's value field is set to the value of the named variable.

If the processing of any variable binding fails for a reason other than listed above, then the Response-PDU is re-formatted with the same values in its request-id and variable-bindings fields as the received GetRequest-PDU, with the value of its error-status field set to 'genErr', and the value of its error-index field is set to the index of the failed variable binding .

Otherwise, the value of the Response-PDU's error-status field is set to 'noError', and the value of its error-index field is zero.

The generated Response-PDU is then encapsulated into a message. If the size of the resultant message is less than or equal to both a local constraint and the maximum message size of the request's source party, it is transmitted to the originator of the GetRequest-PDU.

Otherwise, an alternate Response-PDU is generated. This alternate Response-PDU is formatted with the same value in its request-id field as the received GetRequest-PDU, with the value of its error-status field set to 'tooBig', the value of its error-index field set to zero, and an empty variablebindings field. This alternate Response-PDU is then encapsulated into a message. If the size of the resultant message is less than or equal to both a local constraint and the maximum message size of the request's source party, it is transmitted to the originator of the GetRequest-PDU. Otherwise, the resultant message is discarded. 


\subsubsection{The GetNextRequest-PDU}

A GetNextRequest-PDU is generated and transmitted at the request of a SNMPv2 application.

Upon receipt of a GetNextRequest-PDU, the receiving SNMPv2 entity processes each variable binding in the variable-binding list to produce a Response-PDU. All fields of the ResponsePDU have the same values as the corresponding fields of the received request except as indicated below. Each variable binding is processed as follows:

(1) The variable is located which is in the lexicographically ordered list of the names of all variables which are accessible by this request and whose name is the first lexicographic successor of the variable binding's name in the incoming GetNextRequest-PDU. The corresponding variable binding's name and value fields in the Response-PDU are set to the name and value of the located variable.

(2) If the requested variable binding's name does not lexicographically precede the name of any variable accessible by this request, i.e., there is no lexicographic successor, then the corresponding variable binding produced in the Response-PDU has its value field set to 'endofMibView', and its name field set to the variable binding's name in the request.

If the processing of any variable binding fails for a reason other than listed above, then the Response-PDU is re-formatted with the same values in its request-id and variable-bindings fields as the received GetNextRequest-PDU, with the value of its error-status field set to 'genErr', and the value of its error-index field is set to the index of the failed variable binding.

Otherwise, the value of the Response-PDU's error-status field is set to 'noError', and the value of its error-index field is zero.

The generated Response-PDU is then encapsulated into a message. If the size of the resultant message is less than or equal to both a local constraint and the maximum message size of the request's source party, it is transmitted to the 
originator of the GetNextRequest-PDU.

Otherwise, an alternate Response-PDU is generated. This alternate Response-PDU is formatted with the same values in its request-id field as the received GetNextRequest-PDU, with the value of its error-status field set to 'tooBig', the value of its error-index field set to zero, and an empty variablebindings field. This alternate Response-PDU is then encapsulated into a message. If the size of the resultant message is less than or equal to both a local constraint and the maximum message size of the request's source party, it is transmitted to the originator of the GetNextRequest-PDU. Otherwise, the resultant message is discarded.

\subsubsection{Example of Table Traversal}

An important use of the GetNextRequest-PDU is the traversal of conceptual tables of information within a MIB. The semantics of this type of request, together with the method of identifying individual instances of objects in the MIB, provides access to related objects in the MIB as if they enjoyed a tabular organization.

In the protocol exchange sketched below, a SNMPv2 application retrieves the media-dependent physical address and the address-mapping type for each entry in the IP net-to-media Address Translation Table [9] of a particular network element. It also retrieves the value of sysUpTime [9], at which the mappings existed. Suppose that the agent's IP net-to-media table has three entries:

Interface-Number Network-Address Physical-Address Type

$\begin{array}{lccl}1 & 10.0 .0 .51 & 00: 00: 10: 01: 23: 45 & \text { static } \\ 1 & 9.2 .3 .4 & 00: 00: 10: 54: 32: 10 & \text { dynamic } \\ 2 & 10.0 .0 .15 & 00: 00: 10: 98: 76: 54 & \text { dynamic }\end{array}$

The SNMPv2 entity acting in a manager role begins by sending a GetNextRequest-PDU containing the indicated OBJECT IDENTIFIER values as the requested variable names:

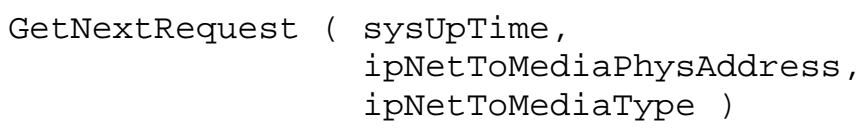


The SNMPV2 entity acting in an agent role responds with a Response-PDU:

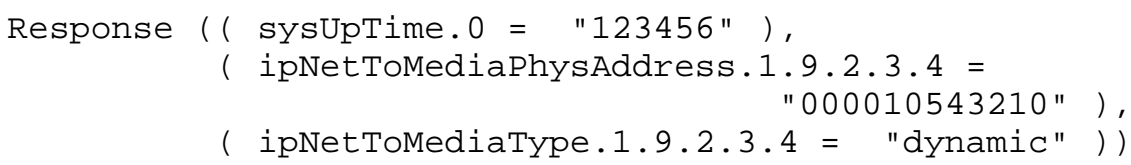

The SNMPV2 entity acting in a manager role continues with:

GetNextRequest ( sysUpTime, ipNetToMediaPhysAddress.1.9.2.3.4, ipNet ToMediaType.1.9.2.3.4)

The SNMPV2 entity acting in an agent role responds with:

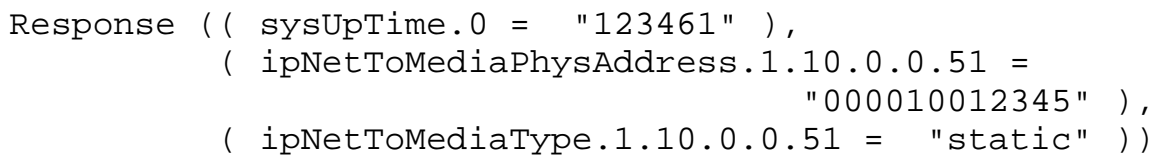

The SNMPV2 entity acting in a manager role continues with:

GetNextRequest ( sysupTime, ipNet ToMediaPhysAddress.1.10.0.0.51, ipNetToMediaType.1.10.0.0.51)

The SNMPv2 entity acting in an agent role responds with:

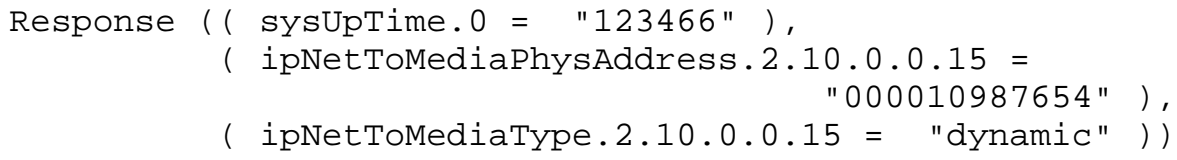

The SNMPV2 entity acting in a manager role continues with:

GetNextRequest ( sysupTime, ipNet ToMediaPhysAddress.2.10.0.0.15, ipNetToMediaType.2.10.0.0.15)

As there are no further entries in the table, the SNMPv2 entity acting in an agent role responds with the variables that are next in the lexicographical ordering of the accessible object names, for example: 


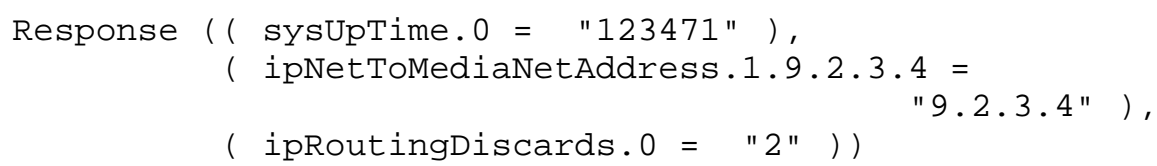

This response signals the end of the table to the SNMPv2 entity acting in a manager role.

\subsubsection{The GetBulkRequest-PDU}

A GetBulkRequest-PDU is generated and transmitted at the request of a SNMPV2 application. The purpose of the GetBulkRequest-PDU is to request the transfer of a potentially large amount of data, including, but not limited to, the efficient and rapid retrieval of large tables.

Upon receipt of a GetBulkRequest-PDU, the receiving SNMPv2 entity processes each variable binding in the variable-binding list to produce a Response-PDU with its request-id field having the same value as in the request. Processing begins by examining the values in the non-repeaters and max-repetitions fields. If the value in the non-repeaters field is less than zero, then the value of the field is set to zero. Similarly, if the value in the max-repetitions field is less than zero, then the value of the field is set to zero.

For the GetBulkRequest-PDU type, the successful processing of each variable binding in the request generates zero or more variable bindings in the Response-PDU. That is, the one-toone mapping between the variable bindings of the GetRequestPDU, GetNextRequest-PDU, and SetRequest-PDU types and the resultant Response-PDUs does not apply for the mapping between the variable bindings of a GetBulkRequest-PDU and the resultant Response-PDU.

The values of the non-repeaters and max-repetitions fields in the request specify the processing requested. One variable binding in the Response-PDU is requested for the first $N$ variable bindings in the request and $\mathrm{M}$ variable bindings are requested for each of the $\mathrm{R}$ remaining variable bindings in the request. Consequently, the total number of requested variable bindings communicated by the request is given by $\mathrm{N}+(\mathrm{M} \star \mathrm{R})$, where $\mathrm{N}$ is the minimum of: a) the value of the non-repeaters field in the request, and b) the number of variable bindings 
in the request; $M$ is the value of the max-repetitions field in the request; and $\mathrm{R}$ is the maximum of: a) number of variable bindings in the request $-\mathrm{N}$, and b) zero.

The receiving SNMPv2 entity produces a Response-PDU with up to the total number of requested variable bindings communicated by the request. The request-id shall have the same value as the received GetBulkRequest-PDU.

If $\mathrm{N}$ is greater than zero, the first through the (N)-th variable bindings of the Response-PDU are each produced as follows :

(1) The variable is located which is in the lexicographically ordered list of the names of all variables which are accessible by this request and whose name is the first lexicographic successor of the variable binding's name in the incoming GetBulkRequest-PDU. The corresponding variable binding's name and value fields in the Response-PDU are set to the name and value of the located variable.

(2) If the requested variable binding's name does not lexicographically precede the name of any variable accessible by this request, i.e., there is no lexicographic successor, then the corresponding variable binding produced in the Response-PDU has its value field set to 'endofMibView', and its name field set to the variable binding's name in the request.

If $M$ and $R$ are non-zero, the $(N+1)$-th and subsequent variable bindings of the Response-PDU are each produced in a similar manner. For each iteration $i$, such that $i$ is greater than zero and less than or equal to $M$, and for each repeated variable, $r$, such that $r$ is greater than zero and less than or equal to $\mathrm{R}$, the $(\mathrm{N}+((\mathrm{i}-1) * \mathrm{R})+r)$-th variable binding of the Response-PDU is produced as follows:

(1) The variable which is in the lexicographically ordered list of the names of all variables which are accessible by this request and whose name is the (i)-th lexicographic successor of the $(N+r)$-th variable binding's name in the incoming GetBulkRequest-PDU is located and the variable binding's name and value fields are set to the name and value of the located variable. 
(2) If there is no (i)-th lexicographic successor, then the corresponding variable binding produced in the ResponsePDU has its value field set to 'endOfMibView', and its name field set to either the last lexicographic successor, or if there are no lexicographic successors, to the $(\mathrm{N}+\mathrm{r})$-th variable binding's name in the request.

While the maximum number of variable bindings in the Response-PDU is bounded by $\mathrm{N}+(\mathrm{M} * \mathrm{R})$, the response may be generated with a lesser number of variable bindings (possibly zero) for either of two reasons.

(1) If the size of the message encapsulating the Response-PDU containing the requested number of variable bindings would be greater than either a local constraint or the maximum message size of the request's source party, then the response is generated with a lesser number of variable bindings. This lesser number is the ordered set of variable bindings with some of the variable bindings at the end of the set removed, such that the size of the message encapsulating the Response-PDU is approximately equal to but no greater than the minimum of the local constraint and the maximum message size of the request's source party. Note that the number of variable bindings removed has no relationship to the values of $N$, $M$, or $R$.

(2) The response may also be generated with a lesser number of variable bindings if for some value of iteration $i$, such that $i$ is greater than zero and less than or equal to $M$, that all of the generated variable bindings have the value field set to the 'endofMibView'. In this case, the variable bindings may be truncated after the $(N+(i$ * R)) -th variable binding.

If the processing of any variable binding fails for a reason other than listed above, then the Response-PDU is re-formatted with the same values in its request-id and variable-bindings fields as the received GetBulkRequest-PDU, with the value of its error-status field set to 'genErr', and the value of its error-index field is set to the index of the failed variable binding.

Otherwise, the value of the Response-PDU's error-status field is set to 'noError', and the value of its error-index field to zero. 
The generated Response-PDU (possibly with an empty variablebindings field) is then encapsulated into a message. If the size of the resultant message is less than or equal to both a local constraint and the maximum message size of the request's source party, it is transmitted to the originator of the GetBulkRequest-PDU. Otherwise, the resultant message is discarded.

\subsubsection{Another Example of Table Traversal}

This example demonstrates how the GetBulkRequest-PDU can be used as an alternative to the GetNextRequest-PDU. The same traversal of the IP net-to-media table as shown in Section 4.2.2.1 is achieved with fewer exchanges.

The SNMPv2 entity acting in a manager role begins by sending a GetBulkRequest-PDU with the modest max-repetitions value of 2, and containing the indicated OBJECT IDENTIFIER values as the requested variable names:

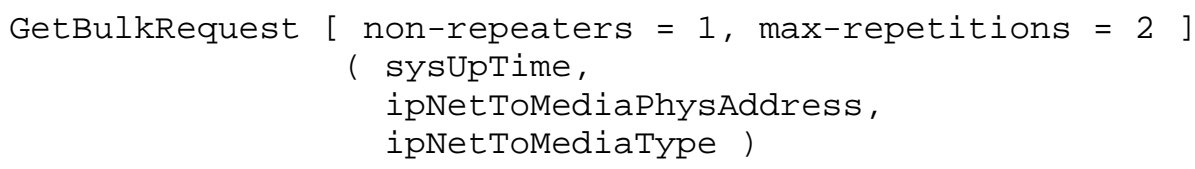

The SNMPV2 entity acting in an agent role responds with a Response-PDU:

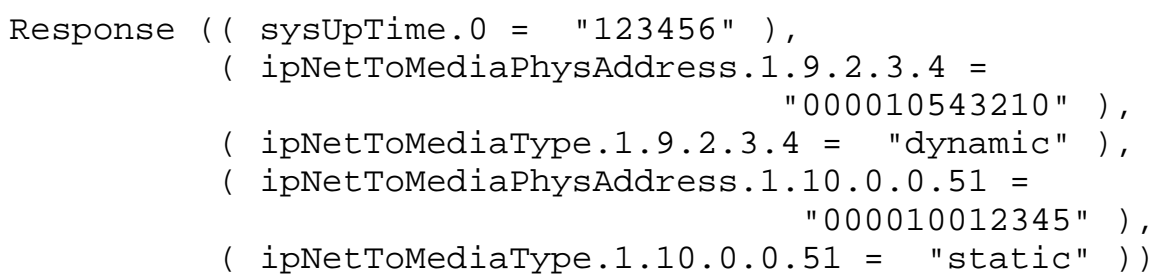

The SNMPv2 entity acting in a manager role continues with:

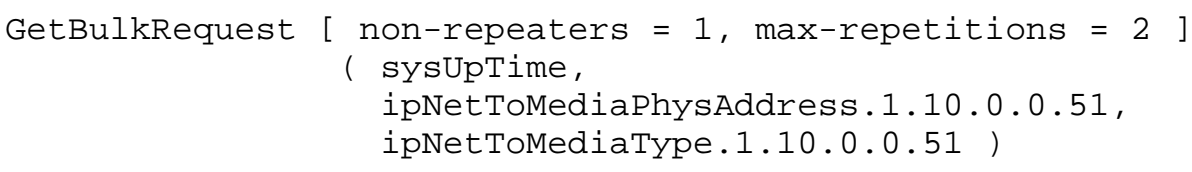


The SNMPV2 entity acting in an agent role responds with:

$$
\begin{aligned}
& \text { Response (( sysUptime.0 = "123466"), } \\
& \text { ( ipNetToMediaPhysAddress.2.10.0.0.15 = } \\
& \text { ( ipNetToMediaType.2.10.0.0.15= } \\
& \text { "000010987654"), } \\
& \text { ( ipNetToMediaNetAddress.1.9.2.3.4 = } \\
& \text { "dynamic" ), } \\
& \text { "9.2.3.4"), } \\
& \text { ( ipRoutingDiscards.0 = "2" )) }
\end{aligned}
$$

This response signals the end of the table to the SNMPv2 entity acting in a manager role.

\subsubsection{The Response-PDU}

The Response-PDU is generated by a SNMPV2 entity only upon receipt of a GetRequest-PDU, GetNextRequest-PDU, GetBulkRequest-PDU, SetRequest-PDU, or InformRequest-PDU, as described elsewhere in this document.

If the error-status field of the Response-PDU is non-zero, the value fields of the variable bindings in the variable binding list are ignored.

If both the error-status field and the error-index field of the Response-PDU are non-zero, then the value of the errorindex field is the index of the variable binding (in the variable-binding list of the corresponding request) for which the request failed. The first variable binding in a request's variable-binding list is index one, the second is index two, etc.

A compliant SNMPV2 entity acting in a manager role must be able to properly receive and handle a Response-PDU with an error-status field equal to 'noSuchName', 'badValue', or 'readonly'. (See Section 3.1.2 of [10].)

Upon receipt of a Response-PDU, the receiving SNMPv2 entity presents its contents to the SNMPV2 application which generated the request with the same request-id value. 


\subsubsection{The SetRequest-PDU}

A setRequest-PDU is generated and transmitted at the request of a SNMPv2 application.

Upon receipt of a SetRequest-PDU, the receiving SNMPV2 entity determines the size of a message encapsulating a Response-PDU with the same values in its request-id, error-status, errorindex and variable-bindings fields as the received SetRequest-PDU. If the determined message size is greater than either a local constraint or the maximum message size of the request's source party, then an alternate Response-PDU is generated, transmitted to the originator of the SetRequestPDU, and processing of the setRequest-PDU terminates immediately thereafter. This alternate Response-PDU is formatted with the same values in its request-id field as the received SetRequest-PDU, with the value of its error-status field set to 'tooBig', the value of its error-index field set to zero, and an empty variable-bindings field. This alternate Response-PDU is then encapsulated into a message. If the size of the resultant message is less than or equal to both a local constraint and the maximum message size of the request's source party, it is transmitted to the originator of the SetRequest-PDU. Otherwise, the resultant message is discarded. Regardless, processing of the setRequest-PDU terminates.

Otherwise, the receiving SNMPv2 entity processes each variable binding in the variable-binding list to produce a ResponsePDU. All fields of the Response-PDU have the same values as the corresponding fields of the received request except as indicated below.

The variable bindings are conceptually processed as a two phase operation. In the first phase, each variable binding is validated; if all validations are successful, then each variable is altered in the second phase. Of course, implementors are at liberty to implement either the first, or second, or both, of the these conceptual phases as multiple implementation phases. Indeed, such multiple implementation phases may be necessary in some cases to ensure consistency.

The following validations are performed in the first phase on each variable binding until they are all successful, or until one fails: 
(1) If the variable binding's name specifies a variable which is not accessible by this request, then the value of the Response-PDU's error-status field is set to 'noAccess', and the value of its error-index field is set to the index of the failed variable binding.

(2) Otherwise, if the variable binding's name specifies a variable which does not exist and could not ever be created, then the value of the Response-PDU's errorstatus field is set to 'nocreation', and the value of its error-index field is set to the index of the failed variable binding.

(3) Otherwise, if the variable binding's name specifies a variable which exists but can not be modified no matter what new value is specified, then the value of the Response-PDU's error-status field is set to 'notWritable', and the value of its error-index field is set to the index of the failed variable binding.

(4) Otherwise, if the variable binding's value field specifies, according to the ASN.1 language, a type which is inconsistent with that required for the variable, then the value of the Response-PDU's error-status field is set to 'wrongtype', and the value of its error-index field is set to the index of the failed variable binding.

(5) Otherwise, if the variable binding's value field specifies, according to the ASN.1 language, a length which is inconsistent with that required for the variable, then the value of the Response-PDU's errorstatus field is set to 'wronglength', and the value of its error-index field is set to the index of the failed variable binding.

(6) Otherwise, if the variable binding's value field contains an ASN.1 encoding which is inconsistent with that field's ASN.1 tag, then: the value of the Response-PDU's errorstatus field is set to 'wrongEncoding', and the value of its error-index field is set to the index of the failed variable binding.

(7) Otherwise, if the variable binding's value field specifies a value which could under no circumstances be assigned to the variable, then: the value of the 
Response-PDU's error-status field is set to 'wrongValue', and the value of its error-index field is set to the index of the failed variable binding.

(8) Otherwise, if the variable binding's name specifies a variable which does not exist but can not be created not under the present circumstances (even though it could be created under other circumstances), then the value of the Response-PDU's error-status field is set to 'inconsistentName', and the value of its error-index field is set to the index of the failed variable binding.

(9) Otherwise, if the variable binding's value field specifies a value that could under other circumstances be assigned to the variable, but is presently inconsistent, then the value of the Response-PDU's error-status field is set to 'inconsistentValue', and the value of its error-index field is set to the index of the failed variable binding.

(10) Otherwise, if the assignment of the value specified by the variable binding's value field to the specified variable requires the allocation of a resource which is presently unavailable, then: the value of the ResponsePDU's error-status field is set to 'resourceUnavailable', and the value of its error-index field is set to the index of the failed variable binding.

(11) If the processing of the variable binding fails for a reason other than listed above, then the value of the Response-PDU's error-status field is set to 'genErr', and the value of its error-index field is set to the index of the failed variable binding.

(12) Otherwise, the validation of the variable binding succeeds.

At the end of the first phase, if the validation of all variable bindings succeeded, then:

The value of the Response-PDU's error-status field is set to 'noError' and the value of its error-index field is zero.

For each variable binding in the request, the named variable is created if necessary, and the specified value is assigned 
to it. Each of these variable assignments occurs as if simultaneously with respect to all other assignments specified in the same request. However, if the same variable is named more than once in a single request, with different associated values, then the actual assignment made to that variable is implementation-specific.

If any of these assignments fail (even after all the previous validations), then all other assignments are undone, and the Response-PDU is modified to have the value of its error-status field set to 'commitfailed', and the value of its error-index field set to the index of the failed variable binding.

If and only if it is not possible to undo all the assignments, then the Response-PDU is modified to have the value of its error-status field set to 'undoFailed', and the value of its error-index field is set to zero. Note that implementations are strongly encouraged to take all possible measures to avoid use of either 'commitFailed' or 'undoFailed' - these two error-status codes are not to be taken as license to take the easy way out in an implementation.

Finally, the generated Response-PDU is encapsulated into a message, and transmitted to the originator of the setRequestPDU.

4.2.6. The SNMPv2-Trap-PDU

A SNMPV2-Trap-PDU is generated and transmitted by a SNMPv2 entity acting in an agent role when an exceptional situation occurs.

The destination(s) to which a SNMPV2-Trap-PDU is sent is determined by consulting the aclTable [5] to find all entries satisfying the following conditions:

(1) The value of aclsubject refers to the SNMPv2 entity.

(2) The value of aclPrivileges allows for the SNMPv2-TrapPDU.

(3) aclResources refers to a SNMPv2 context denoting local object resources, and the notification's administratively assigned name is present in the corresponding MIB view. 
(That is, the set of entries in the viewTable [5] for which the instance of viewIndex has the same value as the aclResources's contextViewIndex, define a MIB view which contains the notification's administratively assigned name.)

(4) If the OBJECTS clause is present in the invocation of the corresponding NOTIFICATION-TYPE macro, then the correspondent variables are all present in the MIB view corresponding to aclResource.

Then, for each entry satisfying these conditions, a SNMPv2Trap-PDU is sent from aclSubject with context aclResources to aclTarget. The instance of snmpTrapNumbers [11] corresponding to aclTarget is incremented, and is used as the request-id field of the SNMPV2-Trap-PDU. Then, the variable-bindings field are constructed as:

(1) The first variable is sysUpTime.0 [9].

(2) The second variable is snmpTrapoID.0 [11], which contains the administratively assigned name of the notification.

(3) If the OBJECTS clause is present in the invocation of the corresponding NOTIFICATION-TYPE macro, then each corresponding variable is copied, in order, to the variable-bindings field.

(4) At the option of the SNMPv2 entity acting in an agent role, additional variables may follow in the variablebindings field.

4.2.7. The InformRequest-PDU

An InformRequest-PDU is generated and transmitted at the request an application in a SNMPv2 entity acting in a manager role, that wishes to notify another application (in a SNMPv2 entity also acting in a manager role) of information in the MIB View of a party local to the sending application.

The destination(s) to which an InformRequest-PDU is sent is determined by inspecting the snmpEventNotifyTable [12], or as specified by the requesting application. The first two variable bindings in the variable binding list of an 
InformRequest-PDU are sysUpTime.0 [9] and snmpEventID.i respectively. If the OBJECTS clause is present in the invocation of the corresponding NOTIFICATION-TYPE macro, then each corresponding variable, as instantiated by this notification, is copied, in order, to the variable-bindings field.

Upon receipt of an InformRequest-PDU, the receiving SNMPv2 entity determines the size of a message encapsulating a Response-PDU with the same values in its request-id, errorstatus, error-index and variable-bindings fields as the received InformRequest-PDU. If the determined message size is greater than either a local constraint or the maximum message size of the request's source party, then an alternate Response-PDU is generated, transmitted to the originator of the InformRequest-PDU, and processing of the InformRequest-PDU terminates immediately thereafter. This alternate ResponsePDU is formatted with the same values in its request-id field as the received InformRequest-PDU, with the value of its error-status field set to 'tooBig', the value of its errorindex field set to zero, and an empty variable-bindings field. This alternate Response-PDU is then encapsulated into a message. If the size of the resultant message is less than or equal to both a local constraint and the maximum message size of the request's source party, it is transmitted to the originator of the InformRequest-PDU. Otherwise, the resultant message is discarded. Regardless, processing of the InformRequest-PDU terminates.

Otherwise, the receiving SNMPv2 entity:

(1) presents its contents to the appropriate SNMPv2 application;

(2) generates a Response-PDU with the same values in its request-id and variable-bindings fields as the received InformRequest-PDU, with the value of its error-status field is set to 'noError' and the value of its errorindex field is zero; and

(3) transmits the generated Response-PDU to the originator of the InformRequest-PDU. 


\section{Acknowledgements}

This document is based, in part, on RFC 1157. The mechanism for bulk retrieval is influenced by many experiments, including RFC1187 and also Greg Satz's work on SNMP over TCP.

Finally, the comments of the SNMP version 2 working group are gratefully acknowledged:

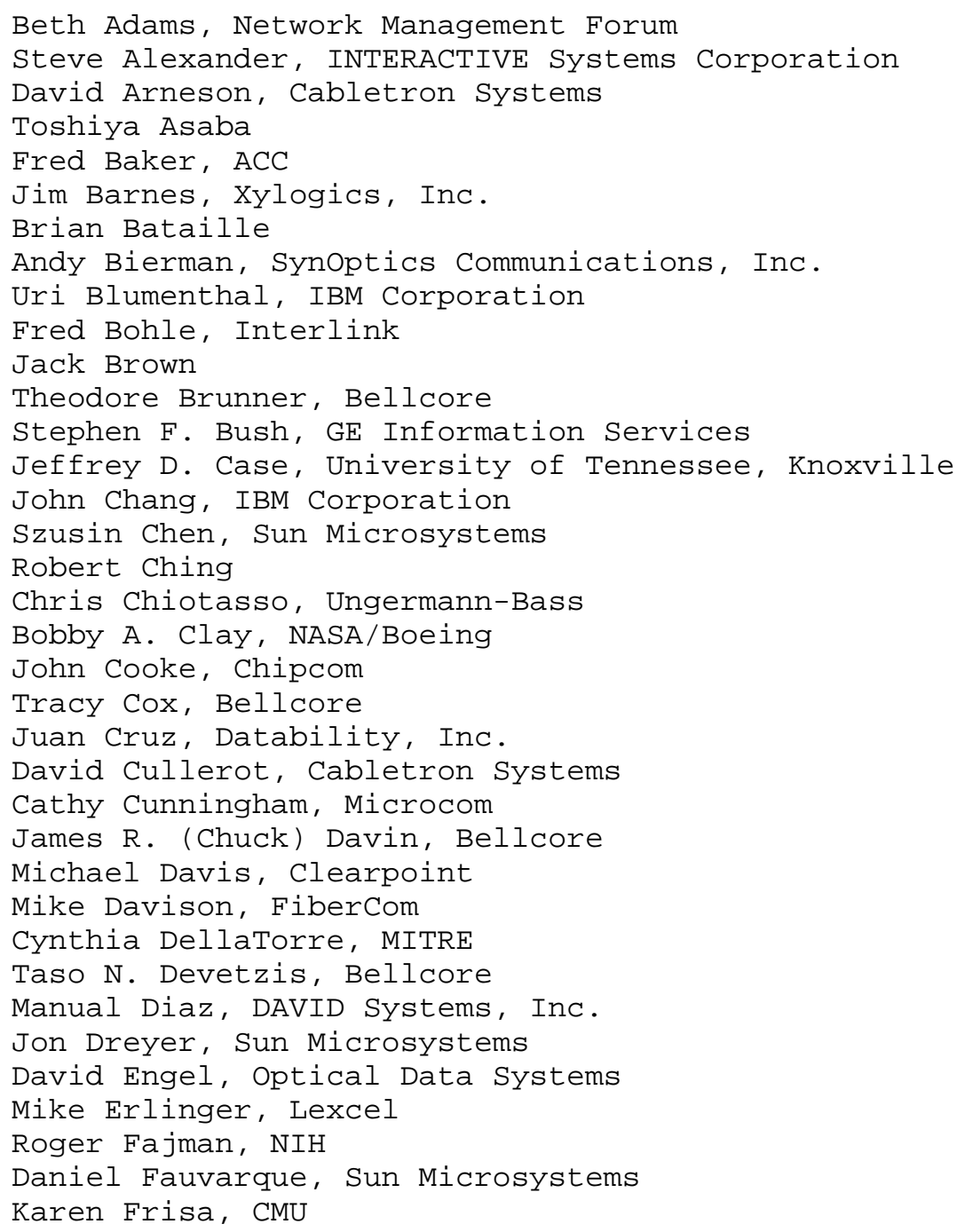




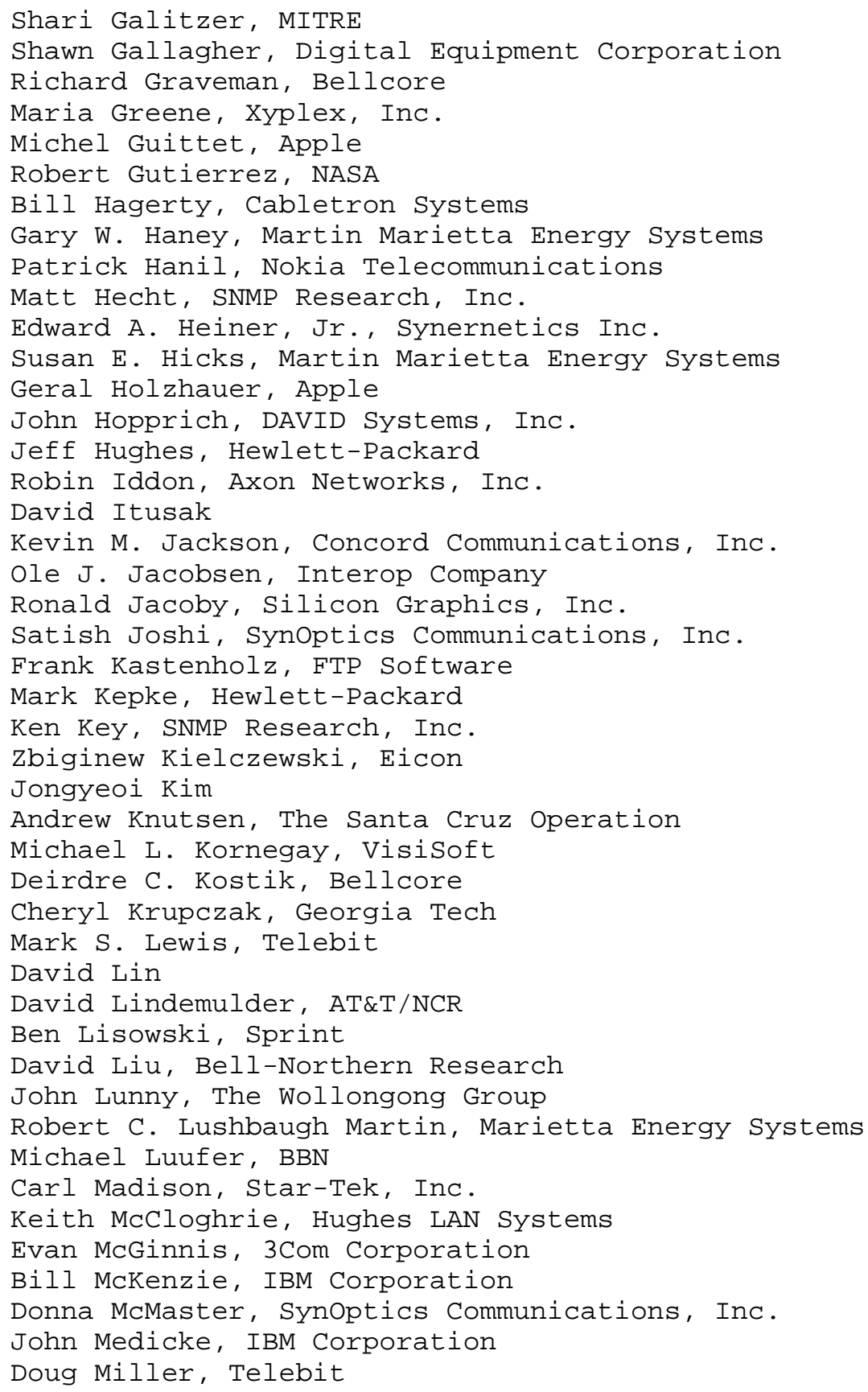




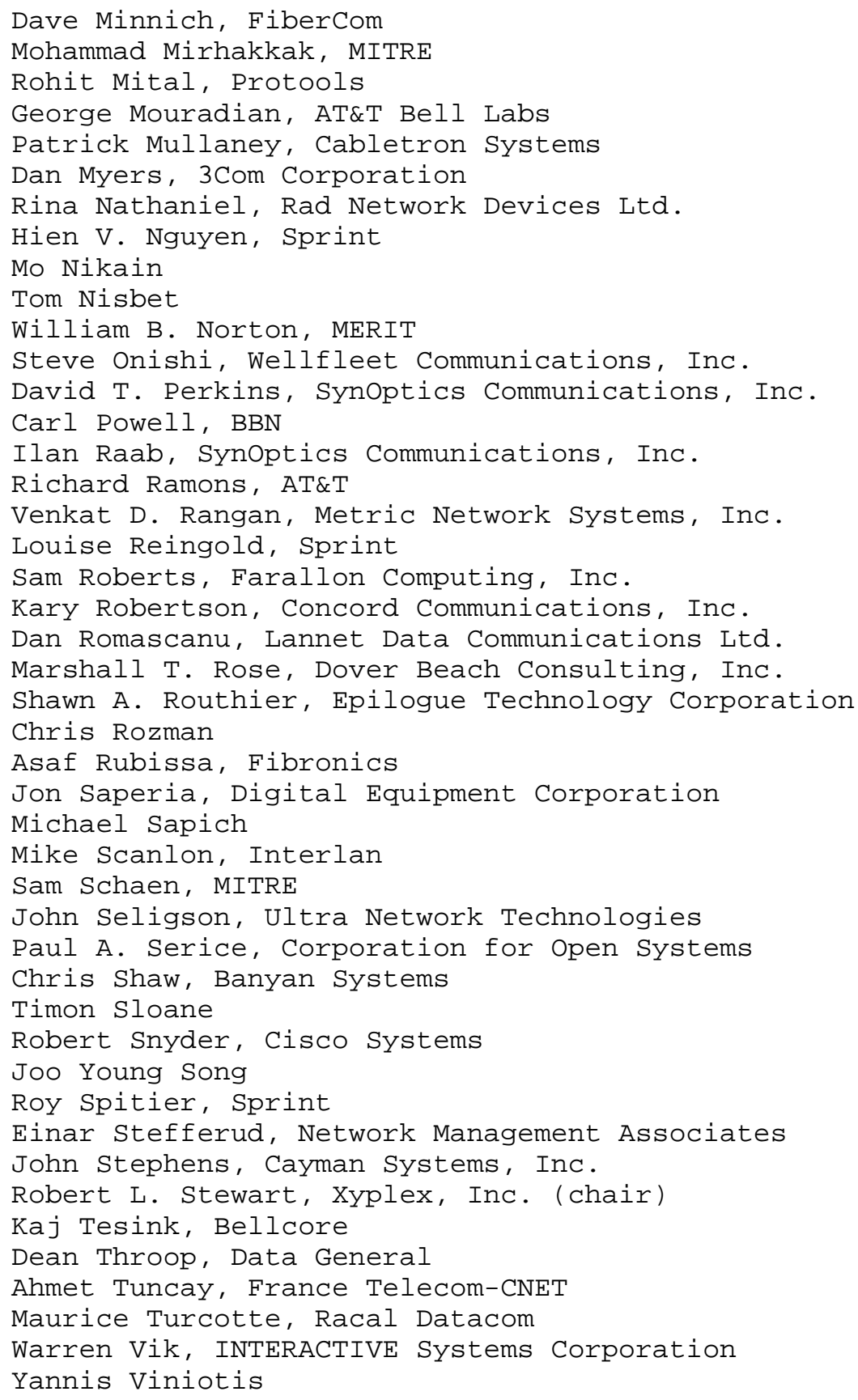




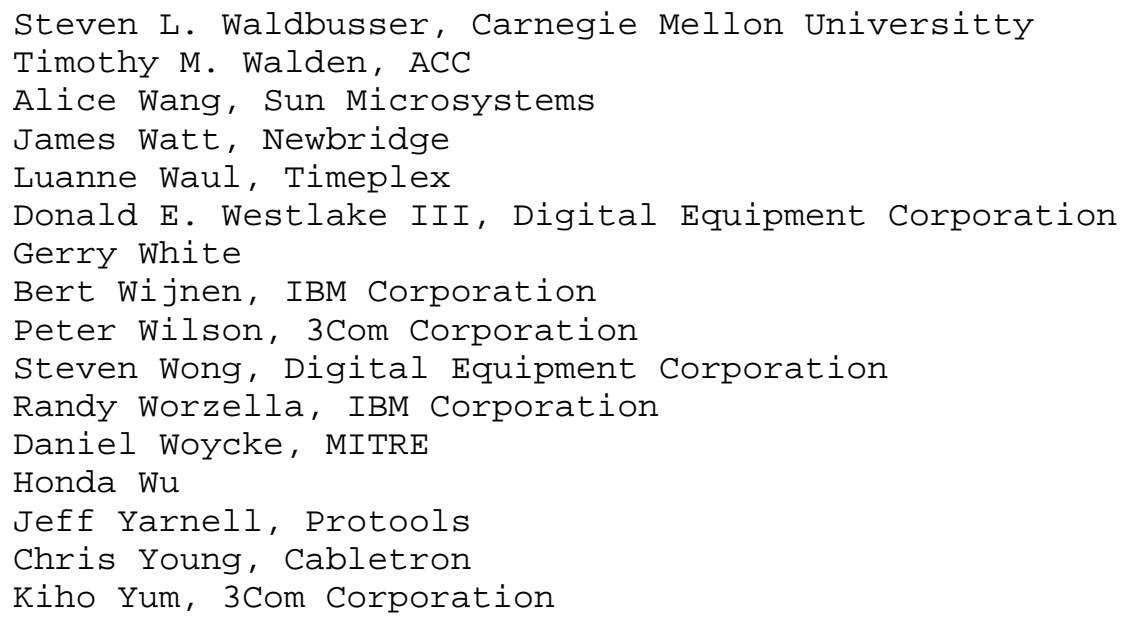


6. References

[1] Information processing systems - Open Systems Interconnection - Specification of Abstract Syntax Notation One (ASN.1), International Organization for Standardization. International Standard 8824, (December, 1987).

[2] Case, J., McCloghrie, K., Rose, M., and Waldbusser, S., "Structure of Management Information for version 2 of the Simple Network Management Protocol (SNMPv2)", RFC 1442, SNMP Research, Inc., Hughes LAN Systems, Dover Beach Consulting, Inc., Carnegie Mellon University, April 1993.

[3] Galvin, J., and McCloghrie, K., "Administrative Model for version 2 of the Simple Network Management Protocol (SNMPv2)", RFC 1445, Trusted Information Systems, Hughes LAN Systems, April 1993.

[4] Case, J., McCloghrie, K., Rose, M., and Waldbusser, S., "Textual Conventions for version 2 of the the Simple Network Management Protocol (SNMPv2)", RFC 1443, SNMP Research, Inc., Hughes LAN Systems, Dover Beach Consulting, Inc., Carnegie Mellon University, April 1993.

[5] McCloghrie, K., and Galvin, J., "Party MIB for version 2 of the Simple Network Management Protocol (SNMPv2)", RFC 1447, Hughes LAN Systems, Trusted Information Systems, April 1993.

[6] C. Kent, J. Mogul, Fragmentation Considered Harmful, Proceedings, ACM SIGCOMM '87, Stowe, VT, (August 1987).

[7] Case, J., McCloghrie, K., Rose, M., and Waldbusser, S., "Transport Mappings for version 2 of the Simple Network Management Protocol (SNMPv2)", RFC 1449, SNMP Research, Inc., Hughes LAN Systems, Dover Beach Consulting, Inc., Carnegie Mellon University, April 1993.

[8] Postel, J., "User Datagram Protocol", STD 6, RFC 768, USC/Information Sciences Institute, August 1980.

[9] McCloghrie, K., and Rose, M., "Management Information Base for Network Management of TCP/IP-based internets: MIB-II", STD 17, RFC 1213, March 1991. 
[10] Case, J., McCloghrie, K., Rose, M., and Waldbusser, S., "Coexistence between version 1 and version 2 of the Internet-standard Network Management Framework", RFC 1452, SNMP Research, Inc., Hughes LAN Systems, Dover Beach Consulting, Inc., Carnegie Mellon University, April 1993.

[11] Case, J., McCloghrie, K., Rose, M., and Waldbusser, S., "Management Information Base for version 2 of the Simple Network Management Protocol (SNMPv2)", RFC 1450, SNMP Research, Inc., Hughes LAN Systems, Dover Beach Consulting, Inc., Carnegie Mellon University, April 1993.

[12] Case, J., McCloghrie, K., Rose, M., and Waldbusser, S., "Manager-to-Manager Management Information Base", RFC 1451, SNMP Research, Inc., Hughes LAN Systems, Dover Beach Consulting, Inc., Carnegie Mellon University, April 1993. 


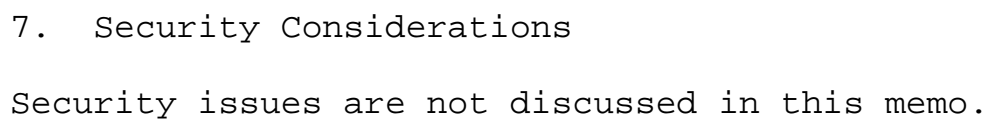

